\title{
Wie können Städte voneinander lernen?
}

\author{
Der Klimaschutz ist keine kommunale Pflicht- \\ aufgabe. Es obliegt den Städten, Lösungen zu \\ finden, die das Klima schützen, sich bewährt \\ haben und keinen Widerstand in der Bevölkerung \\ auslösen. Vernetzung zwischen den Städten \\ ist ein Mittel, um auf erprobte Lösungen zurück- \\ greifen zu können. Von Doris Knoblauch, \\ Arne Riedel und Monica Ridgway
}

D as ökonomische, ökologische und soziale Gleichgewicht einer Stadt steht bei einer schnell wachsenden Bevölkerung unter hohem Druck. Dies trifft insbesondere in Kombination mit den Auswirkungen der Wirtschafts- und Finanzkrise sowie den Folgen des Klimawandels zu. Akteure vor Ort sehen sich großen Herausforderungen und Zielkonflikten gegenüber, wenn sie diesem Druck begegnen wollen. Städtenetzwerke und gemeinsame Forschungsprojekte können Wissen zu bewährten Lösungsansätzen und Umsetzungsstrategien bereitstellen und so die Resilienz einer Stadt unterstützen.

\section{Die Vernetzung von Kommunen im Klimaschutz}

Städte innerhalb der Europäischen Union (EU) können zusätzlich zu Städtenetzwerken auf nationaler Ebene von einem Erfahrungsaustausch zu Herausforderungen, Chancen, Risiken und Positivbeispielen aus der Praxis profitieren. Städte sind Reallabore für sozio-ökonomische und technologische Innovationen, weshalb es wichtig ist, die Ergebnisse bestimmter Politiken und Maßnahmen zu bewerten und Erkenntnisse mit Akteuren zu teilen, die sich ähnlichen Herausforderungen gegenübersehen. Um die Übertragbarkeit verschiedener Herangehensweisen zu erhöhen, sollte die Diversität verschiedener Städte hinsichtlich ihrer geografischen, sozio-ökonomischen, historischen und kulturellen Hintergründe beachtet werden.

Städte haben einen hohen Vernetzungsbedarf im Bereich Klimaschutz, weil dieser nach wie vor keine Pflichtaufgabe ist und in Zeiten angespannter Haushaltslagen das Wissen und der Austausch darüber gefragt sind, welche Lösungsansätze sich bewährt haben und wie Zielkonflikten begegnet werden kann. Kommunen haben deshalb ein Interesse daran,
I sich in vertrautem, kleinen Kreis über Lösungsansätze auszutauschen;

I Ideen präsentiert zu bekommen;

- unkompliziert herauszufinden, wie sie im Vergleich mit anderen Städten dastehen, um sich zu vergewissern, dass sie nichts falsch machen, und um ein Gefühl dafür zu bekommen, wie viel oder wenig sie sich im Vergleich mit anderen engagieren.

Der Artikel basiert auf zwei Projekten. Erstens der wissenschaftlichen Begleitung der Masterplan-Kommunen, die das Ecologic Institut gemeinsam mit Dr. Hans-Joachim Ziesing von 2012 bis 2015 durchgeführt hat, sowie einem EU-Projekt, bei dem unter anderem mehrere europäische Städte dabei begleitet werden, einen Fahrplan für eine $\mathrm{CO}_{2}$-neutrale Zukunft $\mathrm{zu}$ entwickeln. In beiden Projekten gibt es keine offiziellen Netzwerke. Der informelle Austausch zwischen den jeweiligen Akteuren ist jedoch von zentraler Wichtigkeit.

\section{Masterplan-Kommunen in Deutschland}

Während Kommunen bereits seit 2008 Unterstützung vom Bundesministerium für Umwelt, Naturschutz, Bau und Reaktorsicherheit (BMUB, damals noch BMU) für die Erstellung von Klimaschutzkonzepten und für Klimaschutzmanager(innen) beantragen können, erfolgt eine Unterstützung von Vorreiter-Kommunen im Programm „Masterplan 100\% Klimaschutz" erst seit 2012. Dafür wurden 19 Kommunen (Gemeinden, Städte und drei Landkreise) ausgewählt, die sich besonders ambitionierte Klimaschutzziele, nämlich eine Reduktion von $95 \%$ der $\mathrm{CO}_{2}$-Emissionen und eine Einsparung von $50 \%$ des Energieverbrauchs bis 2050, gesetzt haben. Die wissenschaftliche Begleitung und Vernetzung der MasterplanKommunen erfolgt durch ein Konsortium von Instituten und verfolgt vor allem zwei Vernetzungsaspekte:

I Die Rückkoppelung der Kommunen an den Fördermittelgeber BMUB und Projektträger Jülich und

I die Vernetzung der Kommunen untereinander.

Kommunen stehen einem organisierten Austausch grundsätzlich offen gegenüber (Andersen et al. 2010). Tendenziell treten jedoch nur die besonders engagierten Kommunen förmlichen Netzwerken bei, da die Hürden mit einem notwendigen Stadtratsbeschluss und den erforderlichen Mitgliedsbeiträgen relativ hoch sind (Kern et al. 2005).

Die Vernetzung der Masterplan-Kommunen im Rahmen der wissenschaftlichen Begleitung erfolgt insbesondere durch insgesamt acht Vernetzungstreffen zwischen Dezember 2012 


\section{„In einem geschützten Raum \\ können kommunale Akteure \\ ihre Erfahrungen \\ offen diskutieren und so \\ einen Wissensaustausch \\ anstoßen."}

und Mai 2015. Bereits auf dem ersten Treffen wurde deutlich, dass zu Beginn des Masterplan-Vorhabens ein erheblicher Bedarf an intensiverer Vernetzung zwischen den Kommunen bestand. Für die Zeit der Erstellung des Masterplans (im Folgenden als Phase I bezeichnet) rückten insbesondere erforderliche Inhalte und Gestaltung der Masterpläne, die Bilanzierung von Treibhausgasemissionen auf der kommunalen Ebene sowie die Ausgestaltung von Beteiligungsprozessen als Themen in den Vordergrund.

\section{Identifikation von Hemmnissen}

Um diesem Bedarf gerecht zu werden, wurde die Frequenz des Austauschs insbesondere in Phase I gegenüber der Planung deutlich erhöht. Damit ergaben sich auch ab dem zweiten Treffen weitere Zielsetzungen, die den Charakter von einer allgemeinen Vernetzung hin zu einem kooperativen Arbeitstreffen verschoben. Thematische Schwerpunkte lagen zunächst auf:

】 dem Austausch zu gemeinsamen Herausforderungen und Hemmnissen,

- der Identifikation und Definition von Aufgaben des Masterplan-Managements und

I der Erarbeitung gemeinsamer Problemlösungsstrategien. Die Arbeitstreffen während der Erstellungsphase des Masterplans legten den Fokus des Austauschs darauf, den Unterschieden der Masterplan-Kommunen Rechnung zu tragen, indem der Austausch in thematischen oder nach Kommunenstruktur aufgeteilten Arbeitsgruppen stattfand. Die thematische Ausrichtung ermöglichte, Erfahrungen aller Kommunen zu definierten Themen, wie beispielsweise Stakeholder-Beteiligung in der Kommune, Nutzung von Indikatoren oder Finanzierung von Maßnahmen, in den Austausch einzubringen. Die Aufteilung nach der Struktur oder nach der Größe der Masterplan-Kommunen ermöglichte es zum Beispiel, spezifische Herausforderungen für Landkreise oder kleine Kommunen mit einzelnen Masterplan-Managern (anstelle größerer Masterplan-Teams) zu identifizieren und zu diskutieren.
Der sehr aktive Austausch gab Gelegenheit, unterschiedliche Herangehensweisen und gute Beispiele herauszuarbeiten. So wurde von den Masterplan-Kommunen ein weitgehend einheitliches Vorgehen für den erforderlichen Ratsbeschluss zum Masterplan gewählt. Weitere wesentliche Aspekte des Austauschs waren:

- Akteursbeteiligung in Kommune und Kommunalverwaltung,

- Möglichkeiten in der Öffentlichkeitsarbeit,

- Monitoring von Fortschritten und geeignete Indikatoren sowie

I Finanzierungsmodelle für Einzelmaßnahmen und Fördermöglichkeiten auf Landes-, Bundes- und europäischer Ebene.

\section{Austausch von erfolgreichen Ansätzen}

Die Vernetzung der Masterplan-Kommunen führte konkret bereits zu einem vor allem regionalen Austausch zu Herangehensweisen sowie zu einer Übernahme von spezifischen Lösungsansätzen anderer Masterplan-Kommunen. Teilweise erfolgte eine Anpassung von Maßnahmen auf die eigene Kommune, teilweise aber auch eine quasi direkte Übernahme von Ideen, die sich bereits in einer anderen Kommune bewährt hatten, wie zum Beispiel eine Maßnahme der Öffentlichkeitsarbeit zur Suche nach den ältesten Stromfressern in Privathaushalten.

Wie Hintergrundgesprächen mit einzelnen Akteuren entnommen werden konnte, stellte das geschaffene Netzwerk auch eine willkommene Gelegenheit für neu hinzugekommene Personen im Masterplan-Management dar, sich schnell einen Überblick über bestehende Aktivitäten vergleichbarer Kommunen zu verschaffen und sich von den zahlreichen Maßnahmen und Ansätzen inspirieren zu lassen.

Die geplante Ausweitung des Netzwerkes der MasterplanKommunen ab 2016 wird die Begleitung vor neue Herausforderungen stellen, sowohl hinsichtlich der steigenden Anzahl an Kommunenvertreter(inne) $\mathrm{n}$ als auch des Wissenstransfers auf weitere Kommunen. Ansätze könnten dabei die Bildung eines Erfahrungspools aus den ersten Masterplan-Kommunen umfassen, auf den die nächsten Kommunen zugreifen können. Der persönliche Austausch auf Vernetzungstreffen könnte Masterplan-Kommunen zum Beispiel unter regionalen oder thematischen Kriterien zusammenbringen. Zudem ist auch die Ausweitung der Nutzung einer Onlineplattform geplant, die zwischen den Vernetzungstreffen einen regelmäßigen Austausch ermöglichen könnte.

\section{Klimaschutz-Kommunen in Europa}

Auch auf Europäischer Ebene vernetzen sich Vorreiter-Kommunen zum Thema Klimaschutz. Im Projekt „Post-Carbon Cities of Tomorrow“ stehen acht Städte im Zentrum. Barcelona, Istanbul, Kopenhagen und Malmö, Lissabon, Litoměřice, 
Mailand und Turin, Rostock sowie Zagreb wollen jeweils bis $2050 \mathrm{CO}_{2}$-neutral werden.

Das Projekt zielt darauf ab, verschiedenste lokale Stakeholder aktiv einzubinden und den Wissensaustausch zwischen den Städten zu fördern. Im Zentrum des Projektes stehen mehrere partizipative Stakeholder-Workshops in den jeweiligen Städten, auf denen eine gemeinsame $\mathrm{CO}_{2}$-neutrale Vision für 2050 entworfen wird. Darauf aufbauend wird ein Fahroder Aktionsplan entwickelt, wie diese Vision erreicht werden soll. Bei den einzelnen Workshops werden aktuelle Erfolge und Herausforderungen, denen sich die jeweilige Stadt gegenübersieht, herausgearbeitet und eine Diskussion stadtspezifischer, innovativer Maßnahmen angeregt, die sich auf Erkenntnisse lokaler Erfahrungen und Praktiken stützen.

Zusätzlich zu den Stakeholder-Workshops, zielt das Projekt darauf ab, den Austausch und Dialog zwischen diesen und anderen europäischen und nichteuropäischen Städten zu unterstützen. Dafür werden zwei einwöchige Studienfahrten durchgeführt, die es den Repräsentant(inn)en der Städte ermöglichen, sich persönlich zu treffen und sich über Positivbeispiele aus der Praxis auszutauschen. Die Städte, die dabei besucht werden, weisen regional und kulturell unterschiedliche Hintergründe auf, sodass eine Vielfalt an Beispielen zusammenkommt, von denen die Studienfahrtteilnehmer(innen) lernen können.

Die erste Studienfahrt findet im Frühsommer 2015 in Zentral- und Südosteuropa statt, die zweite wird 2016 nach Nordund Westeuropa führen. Während der ersten Studienfahrt werden die beiden Hauptstädte Wien und Zagreb besucht, ebenso wie die mittelgroßen Städte Maribor und Graz und die kleinen Städte Ivanič-Grad und Güssing und damit drei verschiedene nationale Kontexte. Thematisch werden Partizipation und Bürgerbeteiligung diskutiert (in Zagreb und Ivanič-Grad), Stadtplanung, Mobilität, Umwelt und Luftqualität (in Maribor), Ökobezirke, Energieeffizienz in Gebäuden, Energiewende und die Nutzung lokaler und erneuerbarer Energie (in Graz, Güssing und Wien).

\section{Fazit und Ausblick}

Die Beispiele kommunaler Vernetzung, die diesem Artikel zugrunde liegen, entstammen zwei sehr unterschiedlichen Projekten. In einem wurden 19 Kommunen über drei Jahre hinweg intensiv wissenschaftlich begleitet und deren Vernetzung insbesondere durch acht Arbeitstreffen ermöglicht. Beim zweiten Projekt findet der regelmäßige Austausch auf der Metaebene statt, nämlich zwischen den Wissenschaftler(inne)n, die die acht Städte aus acht Ländern begleiten. Deren Vertreter(innen) können sich auf zwei einwöchigen Studienfahrten intensiv kennenlernen und austauschen und dabei verschiedene bewährte Lösungsansätze direkt vor Ort studieren.

In beiden Fällen gab es ein sehr großes Bedürfnis zu erfahren, wie es die anderen machen und welche Lösungen sich bereits bewährt haben. Die Bereitschaft, neue Wege zu gehen oder Experimente zu wagen, war nur bis zu einem gewissen Grad vorhanden. Es zeigte sich, dass Wissen sehr gerne weitergegeben wird und sich die Städte gerne als Verbündete betrachten, nicht etwa als Konkurrenz.

Entscheidend ist nicht zuletzt die Höhe des Sozialkapitals verstanden als Vertrauensgefüge, gemeinsam geteilte Werte und Verständnis - das es verschiedenen Akteuren erlaubt, gemeinsam auf ein Ziel hinzuarbeiten (Huppé et al. 2012). Dieses erlaubt es den kommunalen Akteuren in geschütztem, meist begrenzten Raum, ihre positiven und negativen Erfahrungen offen zu diskutieren und so die Grundlage für einen echten Wissensaustausch zu schaffen.

\section{Literatur}

Andersen, U./Fischer, S./Kuschke, W./Pfundheller, K./Woyke, W. (2010): Von Städtepartnerschaften zu kommunalen Netzwerken - Kommunale Vernetzung als Motor bürgerschaftlichen Engagements in einer europäischen Öffentlichkeit. Abschlussbericht. Internet: www.europa-zentrum-nrw.de/Bilder/abschlussbericht_al.pdf.

Huppé, G. A./Creech, H./Knoblauch, D. (2012): The Frontiers of Networked Governance. Winnipeg.

Kern, K./Niederhafner, S./Rechlin, S./Wagner, J. (2005): Kommunaler Klimaschutz in Deutschland - Handlungsoptionen, Entwicklung und Perspektiven. Berlin. Internet: www.nbn-resolving.de/ urn:nbn:de:0168-ssoar-196722.

Stein, J./Stren, R. (2001): Knowledge Networks in Global Society: Pathways to Development. In: Networks of Knowledge. Collaborative Innovation in International Learning. S. 3-28.

\section{AUTOREN + KONTAKT}

Doris Knoblauch, Arne Riedel und Monica Ridgway sind wissenschaftliche Mitarbeiter(innen) beim Ecologic Institut in Berlin. Ecologic Institut, Pfalzburger Str. 43/44, 10717 Berlin. Internet: www.ecologic.eu Doris Knoblauch, Tel.: +49 30 86880-160, E-Mail: doris.knoblauch@ecologic.eu

Arne Riedel, Tel.: +49 030 86880-192, E-Mail: arne.riedel@ecologic.eu

Monica Ridgway, Tel.: +49 30 86880-190, E-Mail: monica.ridgway@ecologic.eu
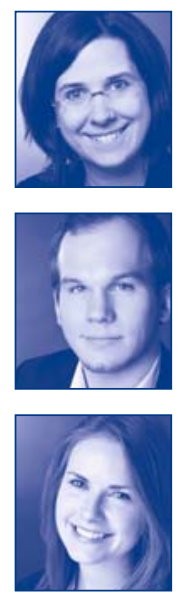